\title{
Reduction in Hospitalizations for Pneumonia Associated With the Introduction of a Pneumococcal Conjugate Vaccination Schedule Without a Booster Dose in Australia
}

\author{
Andrew Jardine, PhD, ${ }^{*}+$ Robert I. Menzies, MPH, ${ }^{*}$ and Peter B. McIntyre, MD, PhD*
}

\begin{abstract}
Background: Postmarketing surveillance of heptavalent pneumococcal conjugate vaccine $(7 \mathrm{vPCV})$ has shown significant reductions in admissions coded as pneumonia in countries where a booster dose is given in the second year of life. In Australia, a 3-dose primary schedule at 2, 4, and 6 months of age without a booster has been funded nationally for nonIndigenous children since 2005 .

Methods: All hospital discharges in Australia with the primary diagnosis coded as pneumonia between July 1998 and June 2007 were identified from a national electronic database. Monthly rates of hospitalization for pneumonia over this period were determined for the age groups $<2,2-4$, $5-17,18-39,40-64$, and $\geq 65$ years. Negative binomial regression modeling, adjusting for background and seasonal trends, was used to quantify the effect of the $7 \mathrm{vPCV}$ program.

Results: A total of 523,591 eligible hospital discharges were identified. In the 30 months following $7 \mathrm{vPCV}$ introduction, there were significant adjusted reductions in all-cause pneumonia in children aged $<2$ and 2 to 4 years of $38 \%$ ( $95 \%$ CI $=36 \%-40 \%)$, and $29 \%(26 \%-31 \%)$, respectively. Reductions of between $3 \%$ and $11 \%$ were observed in the older age groups.

Interpretation: The significant differential effects observed are strongly suggestive of the PCV7 program being responsible for the observed reduction in pneumonia hospitalizations in Australia, and the magnitude was comparable to that documented in countries with a booster dose. This finding appears robust and may be related to high levels of vaccination coverage and catch-up early in the program, or to relatively lower levels of serotype replacement without a booster dose.
\end{abstract}

Key Words: heptavalent pneumococcal conjugate vaccine, pneumonia, hospitalization, immunization schedule

(Pediatr Infect Dis J 2010;29: 000-000)

$\mathrm{P}$ neumonia is a frequent cause of hospitalization at both ends of the age spectrum in Australia ${ }^{1,2}$ and in other developed countries. ${ }^{3-5}$ In developing countries it is the single largest cause of mortality among children less than 5 years of age, conservatively estimated to

Accepted for publication January 20, 2010.

From the *National Centre for Immunisation Research and Surveillance of Vaccine Preventable Diseases, The Children's Hospital at Westmead and the University of Sydney, New South Wales, Australia; and †National Centre for Epidemiology and Population Health, Australian National University, Canberra, Australia.

Supported by Australian Government Department of Health and Ageing, the NSW Department of Health, and The Children's Hospital at Westmead.

NCIRS is a collaborating unit of the Australian Institute of Health and Welfare.

A.J. is a Master of Applied Epidemiology scholar.

Address for correspondence: Andrew Jardine, $\mathrm{PhD}$, National Centre for Epidemiology and Population Health, The Australian National University Canberra, ACT, 0200, Australia. E-mail: u4052357@anu.edu.au.

Supplemental digital content is available for this article. Direct URL citations appear in the printed text and are provided in the HTML and PDF versions of this article on the journal's Web site (www.pidj.com).

Copyright (C) 2010 by Lippincott Williams \& Wilkins

ISSN: 0891-3668/10/2907-0001

DOI: $10.1097 /$ INF.0b013e3181d7d09c cause more than 2 million deaths annually. ${ }^{6}$ Studies in Finland, ${ }^{7}$ the United States, ${ }^{8}$ and Switzerland ${ }^{9}$ have demonstrated Streptococcus pneumoniae to be the most common causative pathogen among children hospitalized for pneumonia $(37 \%, 44 \%$, and $46 \%$, respectively) before use of heptavalent pneumococcal conjugate vaccine (7vPCV). In Australia, a 1989 study demonstrated S. pneumoniae to be the most commonly identified pathogen $(42 \%)$ in hospitalized adults with community-acquired pneumonia. ${ }^{10}$ The overall magnitude was substantially lower at $14 \%$, although $S$. pneumoniae remained the most frequently identified cause of community-acquired pneumonia, in a more recent Australian study of hospitalized adults. ${ }^{11}$ A universally funded $7 \mathrm{vPCV}$ program was commenced in January 2005 in Australia with a 3-dose schedule at 2, 4, and 6 months of age without a booster dose (often referred to as " $3+0$ " schedule), and catch-up immunization was funded for children up to 2 years. ${ }^{12}$

Data supporting the effect of $7 \mathrm{vPCV}$ on hospitalization for pneumonia come from clinical trials and observational studies. The pivotal clinical trial in the United States, using a 3-dose schedule with a booster in the second year of life $(3+1$ schedule), demonstrated a significant reduction in radiologically confirmed pneumonia of $23 \%$ (95\% CI: 9\%-35\%) for children less than 2 years of age, and a lesser reduction in clinically diagnosed pneumonia (7\%; 95\% CI: $0 \%-14 \%)$, which was not statistically significant. ${ }^{13}$ Clinical trials of a nonavalent conjugate vaccine in 2 settings in Africa ${ }^{14,15}$ found that a 3-dose primary schedule without a booster dose was also effective in preventing serotype-specific invasive disease and pneumonia. For invasive disease, postlicensure data from the United States found that a $3+17 \mathrm{vPCV}$ schedule gave significantly higher protection than a $3+0$ schedule against vaccine type infection. ${ }^{16,17}$

All postlicensure data regarding the effect of $7 \mathrm{vPCV}$ on hospitalizations coded as pneumonia published to date have arisen from North American settings with a booster dose. Two studies from the United States demonstrated statistically significant reductions of $39 \%{ }^{18}$ and $52 \%,{ }^{19}$ respectively, in hospitalizations coded as pneumonia for children less than 2 years following $7 \mathrm{vPCV}$ introduction, which appear to have been maintained longer term. ${ }^{20}$ A third study found a significant reduction of $41 \%$ in hospitalizations coded as pneumonia for infants less than 1 year, but not older age groups. ${ }^{21}$ Finally in Quebec, Canada, where a $2+1$ schedule has been recommended since 2004 with a catch-up program up to 5 years of age, an appreciably smaller reduction of $8.2 \%$ was demonstrated in children less than 2 years of age up to March $2006 .^{22}$ The unique $3+0$ schedule used in Australia, and the availability of national level datasets over a long period, provided an opportunity to determine whether a reduction in hospitalizations coded as pneumonia could also be demonstrated in this setting.

\section{METHODS}

\section{Data Sources}

The Australian Institute of Health and Welfare National Hospital Morbidity Database is an electronic collection of de-identified records of episodes of care, which includes more than $99 \%$ of public and private hospitals in Australia. Medical notes are reviewed by 
certified medical archivists after hospital discharge and a primary diagnosis (the condition associated with the highest use of resources) and up to 31 secondary diagnoses are coded in the database according to the 10th Revision of the International Classification of Diseases Australian Modification (ICD-10-AM). ${ }^{23}$ All records with a primary diagnosis code for pneumonia were extracted between July 1998 and June 2007 inclusive. Cases were classified into 3 diagnostic groups based on the methods of De Wals et al. ${ }^{21}$ The first group was presumptive pneumococcal pneumonia, which was defined as all cases where $S$. pneumoniae was coded as the causative pathogen (ICD-10 code: J13) as well as lobar pneumonia (J18.1), as the proportion of $S$. pneumoniae infection is high in such cases. ${ }^{24}$ The second group, unspecified pneumonia, included all hospitalizations coded as pneumonia without a causative organism identified. The remaining hospitalizations, where a pathogen other than S. pneumoniae was specifically coded, were classified as nonpneumococcal pneumonia (for full list of codes and groups see Table, Supplemental Digital Content 1, http://links.lww.com/INF/A421). Indigenous Australians were excluded from the analysis due to differences in vaccine program implementation and background data about the etiology of pneumonia in this group. ${ }^{25,26}$ In accordance with Australian Institute of Health and Welfare guidelines, cases were classified as non-Indigenous when so coded or when Indigenous status was not reported. ${ }^{27}$

\section{Statistical Analysis}

Overall rates per 1000 person years were calculated by dividing the total number of hospitalizations during the 9-year period by the cumulative Australian population and multiplying by 1000. Agespecific rates, standardized to the 2001 Australian population, were also calculated where appropriate.

The data were analyzed as a time series. Monthly rates of all-cause pneumonia hospitalizations per 100,000 population were plotted for each year in the following age groups: $<2$ years; $2-4$ years; 5-17 years; $18-39$ years; $40-64$ years; and $\geq 65$ years. A 12-month moving average was then overlaid to smooth out seasonal variation and clearly display the underlying trend in the data. For each of the aforementioned age groups, the change over time in rates of hospitalizations for all cause, and the 3 diagnostic subgroups of pneumonia, associated with the introduction of the universal 7vPCV program was evaluated using multivariate negative binomial regression in STATA 9.2 (StataCorp, TX, 2007). This regression model was selected for use as goodness-of-fit tests using the Pearson statistic indicated that standard Poisson analysis was inappropriate. The number of cases per month was assigned as the dependent variable and the population estimate for that year as the offset variable. Independent variables included in the model were background trend (1/12 increments per month), seasonality (indicator variables for each calendar month), and a vaccine term. The vaccine term was modeled as a linear increase per month between January and December 2005, to a maximum of $91 \%$ coverage, which approximated the increase in percentage vaccine uptake over this period. ${ }^{28}$ The background trend term was dropped from the model for age groups where it was not a statistically significant component. The vaccine term regression coefficients from the final adjusted analysis were then exponentiated to generate an estimate of the percentage change in pneumonia hospitalization rates associated with vaccine introduction. The model residuals were plotted in a correlogram to check for autocorrelation and ensure the standard errors and $P$ values generated from the model were accurate.

\section{RESULTS}

There were 523,591 recorded hospitalizations for all-cause pneumonia in Australia between July 1998 and June 2007. In 85.1\% of cases, coding did not specify a causative organism. Codes for lobar and pneumococcal pneumonia accounted for 5\% and the remaining $9.9 \%$ was coded as due to other specified pathogens. The rate per 1000 person years varied with age as shown in Table 1 .

\section{All-Cause Pneumonia}

Monthly age-specific rates of hospitalization for pneumonia per 100,000 population are shown in Figure 1. A strong seasonal pattern is evident in all age groups, with the majority of hospitalizations during the southern hemisphere winter and spring months of June to November, inclusive. The 12-month moving average revealed a marked decline for children $<4$ years of age, commenc-

TABLE 1. Hospital Discharges for Pneumonia in Australia, 1998-2007

\begin{tabular}{|c|c|c|c|}
\hline & Number & $\%$ Total All-Cause & Rate per 1000 p/yr* \\
\hline All-cause pneumonia & 523,591 & $100 \%$ & $2.94(2.99)$ \\
\hline \multicolumn{4}{|l|}{ Subgroup } \\
\hline Pneumococcal pneumonia & 26,286 & $5.0 \%$ & $0.15(0.15)$ \\
\hline $\begin{array}{l}\text { Pneumonia, organism unspecified but } \\
\text { excluding lobar pneumonia }\end{array}$ & 445,640 & $85.1 \%$ & $2.50(2.55)$ \\
\hline $\begin{array}{l}\text { Pneumonia, organism specified but excluding } \\
\text { S. pneumoniae }\end{array}$ & 51,655 & $9.9 \%$ & $0.15(0.15)$ \\
\hline \multicolumn{4}{|l|}{ Age group } \\
\hline$<2 \mathrm{yr}$ & 30,277 & $5.8 \%$ & 5.92 \\
\hline $2-4 \mathrm{yr}$ & 30,807 & $5.9 \%$ & 3.95 \\
\hline $5-17$ yr & 30,007 & $5.7 \%$ & 0.85 \\
\hline $18-39 \mathrm{yr}$ & 49,444 & $9.4 \%$ & 0.78 \\
\hline $40-64 \mathrm{yr}$ & 102,997 & $19.7 \%$ & 1.67 \\
\hline$\geq 65 \mathrm{yr}$ & 280,059 & $53.5 \%$ & 11.15 \\
\hline \multicolumn{4}{|l|}{ Gender } \\
\hline Male & 279,048 & $53.3 \%$ & $3.11(3.60)$ \\
\hline Female & 244,534 & $46.7 \%$ & $2.76(2.57)$ \\
\hline Not specified & 9 & $0.0 \%$ & \\
\hline \multicolumn{4}{|l|}{ Season } \\
\hline Spring & 150,765 & $28.8 \%$ & \\
\hline Summer & 105,950 & $20.3 \%$ & \\
\hline Autumn & 97,639 & $18.6 \%$ & \\
\hline Winter & 169,237 & $32.3 \%$ & \\
\hline
\end{tabular}

*Age-adjusted rate standardized to 2001 Australian population shown in parentheses where appropriate. 

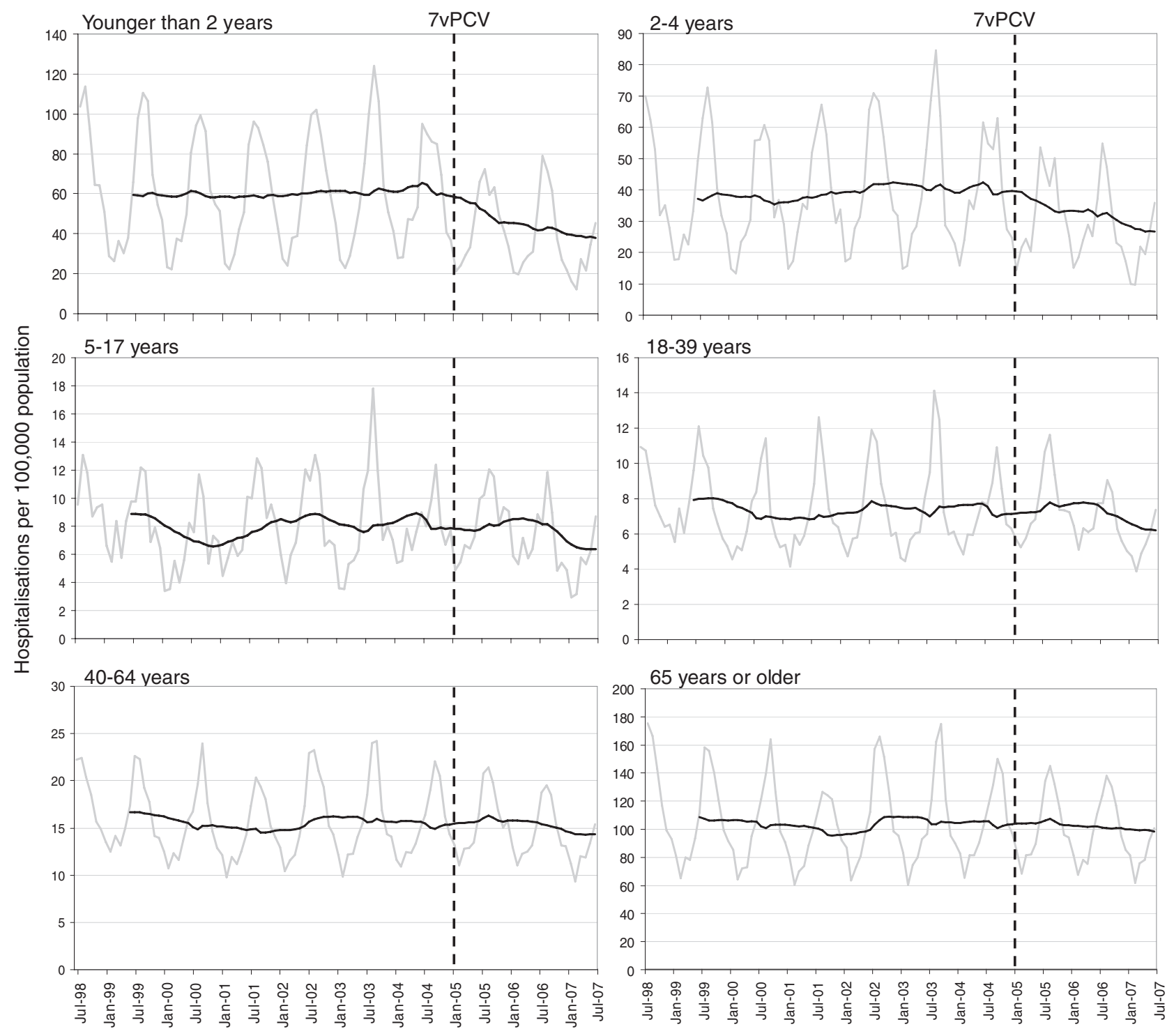

Month of separation

FIGURE 1. Age-specific monthly rates of hospitalization for all cause pneumonia per 100,000 population in Australia, July 1998 to June 2007 (gray line). Solid black line indicates annual moving average for the previous 12 months. Vertical dashed lines represent pneumococcal conjugate vaccine program introduction. Note the variation in the scale on the $y$-axis between age groups.

ing after the $7 \mathrm{VPCV}$ program began in January 2005, which appears greater for those less than 2 years than those 2 to 4 years of age. Changes in older age groups are less evident on inspection but there is some suggestion of a decline in the moving average for all age groups.

Model estimates were validated by the lack of autocorrelation in the residuals of the model for any age group. After adjusting for background trend and seasonal cycle, a statistically significant decrease in age-specific rates of hospitalization in the period following vaccine introduction was observed for all-cause pneumonia in children aged less than 2 years (38\%), and 2 to 4 years $(28 \%)$, as shown in Figure 2. A 3\% to $11 \%$ reduction, of borderline statistical significance, was observed in the older age groups. Overall, the model estimated that for children aged $\leq 4$ years, $7 \mathrm{vPCV}$ prevented approximately 4700 hospitalizations for pneumonia over the 30 -month period from the introduction of universal funding in January 2005.

\section{Pneumonia by Diagnostic Subgroup}

The pattern by diagnostic subgroup was consistent with a vaccine effect. This is based on the observation of an almost $70 \%$ reduction in rates of presumptive pneumococcal pneumonia in children less than 5 years, compared with approximately $40 \%$ for pneumonia of unspecified cause, and no reduction in pneumonia coded as due to other specified organisms (Fig. 3). Although the magnitude of absolute reductions in all-cause coded pneumonia was less in older age groups, the progressive pattern of greater reductions in more 


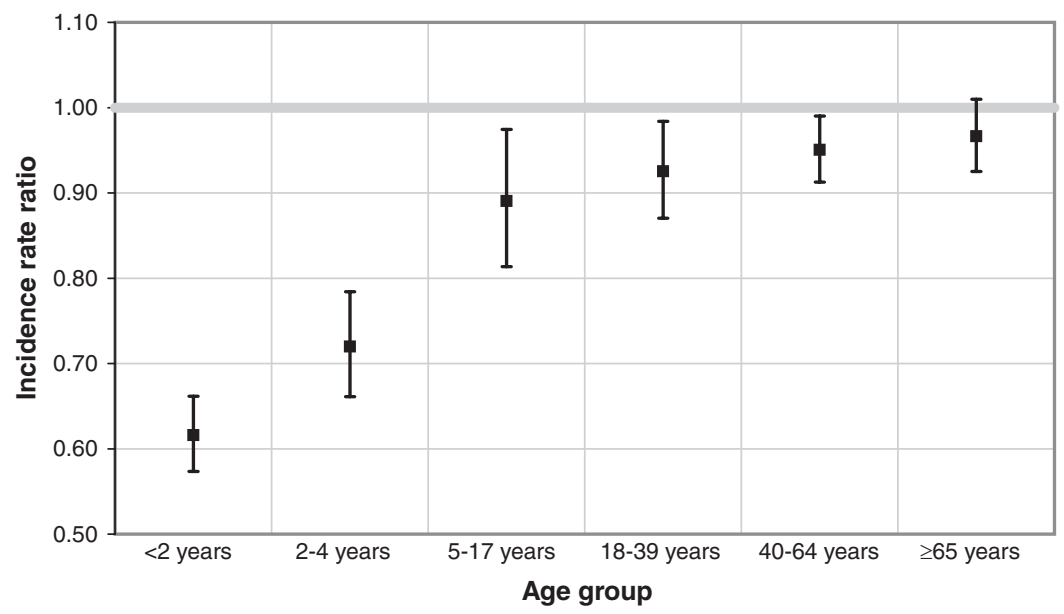

FIGURE 2. Age-specific multivariate negative binomial regression modeling parameter estimates for the 7vPCV program impact on all cause pneumonia, Australia, July 1998 to June 2007 (error bars indicate 95\% confidence intervals).

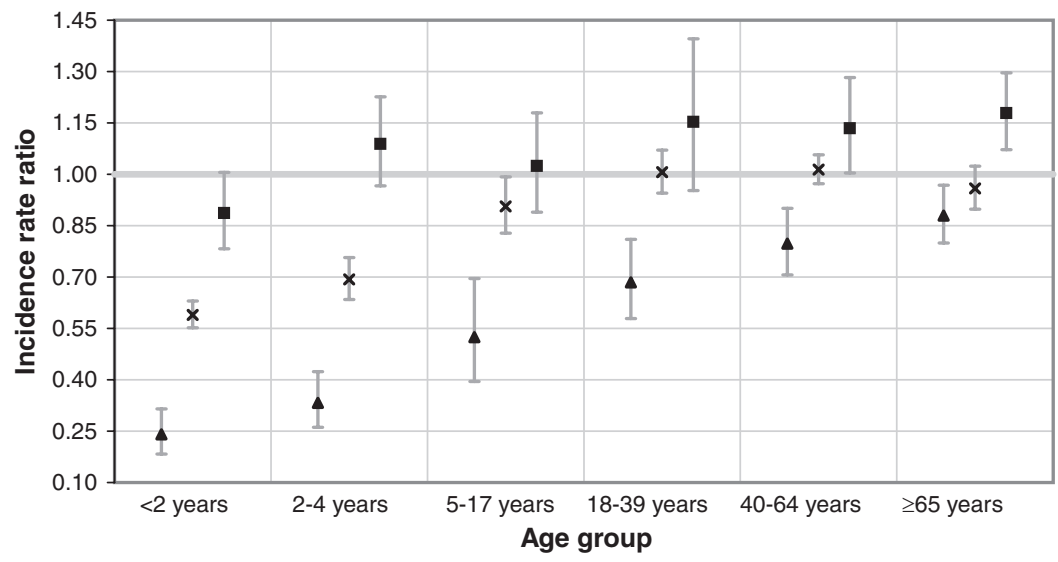

$\Delta$ Pneumoccocal and lobar $\mathbf{x}$ Unspecified cause $\mathbf{O}$ Other specified cause

FIGURE 3. Age-specific multivariate negative binomial regression modeling parameter estimates for the 7vPCV program impact by diagnostic group, Australia, July 1998 to June 2007 (error bars indicate 95\% confidence intervals).

specific coded subgroups remained. It was notable that in adults 40 years and older, although there was a statistically significant increase in cases coded as due to specified causes other than $S$. pneumoniae, relative to background trends, there was nevertheless a statistically significant decrease in all-cause pneumonia.

\section{DISCUSSION}

The Australian $3+0$ schedule for routine administration of $7 \mathrm{vPCV}$ differs from other developed countries, which include a booster dose in the second year of life after either 2 or 3 primary doses $(2+1$ or $3+1) .{ }^{29,30}$ Immunogenicity and carriage studies have suggested that protection against outcomes more closely related to mucosal antibody titers, such as pneumonia and otitis media, may require the higher antibody titers induced by a dose in the second year of life. ${ }^{31,32}$ However, we have previously demonstrated that myringotomy with ventilation tube insertion procedures, used to treat severe otitis media, reduced significantly in children $<2$ years of age following the introduction of the Australian $3+07 \mathrm{vPCV}$ program. ${ }^{33}$

This is the first postlicensure observational study of hospitalizations for pneumonia in a country not using a booster dose of
$7 \mathrm{vPCV}$ in the second year of life. The most marked reduction in the rate of hospitalization for pneumonia was in children $<2$ and 2 to 4 years of age (38\% and $28 \%$, respectively). The decrease seen in children $<2$ years of age was of similar magnitude to that reported from a study of a subset of hospitalizations coded as pneumonia from the United States, with a recommended $3+1$ schedule, in the first year postvaccination and appreciably greater than the $8 \%$ reduction documented in Quebec under a 2+1 schedule. The point estimate for 2- to 4-year olds was also greater than the US estimate of $17 \%(-3 \%$ to $34 \%$ ). Statistically significant decreases were also observed in older, nonvaccinated, age groups. Interpretation of the findings for older age groups must be cautious, however, as statistical significance was marginal and arose from analysis of a large dataset. More importantly, the consistent pattern of different rates of change across age groups and between diagnostic groups supports the trends observed being related to vaccination program implementation rather than nonspecific confounding effects, such as changes in diagnostic coding or clinical practice.

Caution is required when comparing results between countries due to variations in vaccine program implementation, uptake, 
and coverage. In Australia, approximately $91 \%$ of children born during the first year of the program received 3 doses by 12 months of age. ${ }^{34}$ The program also included a catch-up component for children up to 2 years of age in the first year, with an estimated coverage of approximately $60 \% .{ }^{12}$ Coverage of $87 \%$ was achieved in the first year of the program in Quebec and a passive catch-up approach for children between 4 and 59 months of age was estimated to achieve coverage of $73 \%{ }^{35}$ By comparison, when $7 \mathrm{vPCV}$ was introduced in the United States, although catch-up to 2 years of age was also recommended, there was both slower uptake and early vaccine shortages with reduced dosage recommendations. ${ }^{36}$ Finally, while influenza immunization has been recommended in Australia for all persons who wish to reduce their risk of acquiring influenza for some time, during the study period influenza vaccine was funded under the National Immunization Program only for persons more than 65 years and Indigenous persons over 50 years of age. ${ }^{12}$ The low level of coverage in younger age groups would not be expected to have had any influence on hospitalizations for pneumonia.

The primary limitation of this ecologic study is that it is not possible to exclude other external factors that might confound the observed effect of $7 \mathrm{vPCV}$ on the age-specific rates of hospitalization for pneumonia, such as changing management or coding practices for pneumonia. The Pneumonia Severity Index ${ }^{37}$ has been recommended in Australian guidelines since 2003 as a tool to determine which cases of community-acquired pneumonia should be treated as outpatients and which should be admitted to hospital. Clinical audits and studies have demonstrated that compliance with and use of Pneumonia Severity Index varies considerably and is generally low. ${ }^{11,38,39}$ Therefore, it is unlikely that a systematic shift in the management of pneumonia coincided with the introduction of the pneumococcal vaccine. A discharge diagnosis of pneumonia is commonly made on clinical and radiologic findings without microbiologic confirmation, and so a specific causative pathogen is not identified in most cases in Australia and elsewhere. ${ }^{40}$ Improved procedures for diagnostic testing may have influenced the pattern observed between the diagnostic subgroups. This is demonstrated by comparing the percentage of pneumonia cases with unspecified etiology in this study $(85 \%)$ with the $55 \%$ achieved in a recent Australian study where cases hospitalized for community-acquired pneumonia underwent a standardized, detailed assessment for bacterial, and viral pathogens. ${ }^{11}$ It would, therefore, be expected that more cases of pneumococcal pneumonia would be recorded over time, not fewer as we observed, so it is possible the effect observed in this group is actually an underestimate. Regardless, any changes in diagnostic testing procedures should not have affected our primary study outcome of all-cause pneumonia, or resulted in the differential pattern by age evident in the data. ICD-10 coding using all diagnosis codes has been demonstrated to have good sensitivity (98\%) and specificity $(97 \%)$ in recording medical record notation of all-cause pneumonia in the hospitalized elderly in Australia. ${ }^{41}$

We believe that this study adds to information from a previous study on another non-IPD (invasive pneumococcal disease) outcome (myringotomy with ventilation tube insertion procedures) ${ }^{33}$ and provides further evidence to suggest that the effect of the Australian pneumococcal conjugate vaccine program without a booster dose is comparable to that of schedules that include a booster in the second year. The World Health Organization recommends a $3+0$ schedule at 6,10 , and 14 weeks in developing countries where financial and operational barriers prohibit booster dose inclusion. ${ }^{42}$ This abbreviated schedule is cost-effective in such settings based on data from clinical trials. ${ }^{43}$ The current study provides data from a large population over a long period further supporting the effectiveness of a $7 \mathrm{vPCV}$ schedule without a booster dose, which has implications for cost-effectiveness. Population level catch-up programs were found to increase temporarily the effectiveness of a primary schedule without booster for invasive Haemophilus influenzae type b disease in the United Kingdom, ${ }^{44}$ and it is possible that the effectiveness of the Australian pneumococcal vaccination program without a booster may wane over time. Continued monitoring and longitudinal analyses are warranted to assess the longer term effects of various contributing factors such as dosing schedule, serotype replacement, and vaccination coverage as well as catch-up programs. This will be particularly important as new pneumococcal conjugate vaccines offering additional serotype protection are licensed and included in immunization programs.

\section{ACKNOWLEDGMENTS}

The authors acknowledge the Australian Institute of Health and Welfare, Hospitals and Mental Health Services Unit and Population Health Unit, for data from the National Hospital Morbidity Database. Professor William Dunsmuir, Head of School of Mathematics and Statistics at the University of New South Wales, provided valuable assistance with the regression modeling.

\section{REFERENCES}

1. Burgner D, Richmond P. The burden of pneumonia in children: an Australian perspective. Paediatr Respir Rev. 2005;6:94-100.

2. Skull SA, Andrews RM, Byrnes GB, et al. Hospitalized communityacquired pneumonia in the elderly: an Australian case-cohort study. Epidemiol Infect. 2009;137:194-202.

3. Farha T, Thomson AH. The burden of pneumonia in children in the developed world. Paediatr Respir Rev. 2005;6:76-82

4. Vila-Corcoles A, Ochoa-Gondar O, Rodriguez-Blanco T, et al. Epidemiology of community-acquired pneumonia in older adults: a population-based study. Respir Med. 2009;103:309-316.

5. Jackson ML, Neuzil KM, Thompson WW, et al. The burden of communityacquired pneumonia in seniors: results of a population-based study. Clin Infect Dis. 2004;39:1642-1650.

6. Wardlaw T, Johansson EW, Hodge M. Pneumonia: the forgotten killer of children. The United Nations Childrens Fund (UNICEF)/World Health Organisation (WHO), 2006. Available at: http://www.unicef.org/publications files/Pneumonia_The_Forgotten_Killer_of_Children.pdf. Accessed March 18, 2009.

7. Juven T, Mertsola J, Waris M, et al. Etiology of community-acquired pneumonia in 254 hospitalized children. Pediatr Infect Dis J. 2000;19:293298.

8. Michelow IC, Olsen K, Lozano J, et al. Epidemiology and clinical characteristics of community-acquired pneumonia in hospitalized children. Pediatrics. 2004;113:701-707.

9. Cevey-Macherel M, Galetto-Lacour A, Gervaix A, et al. Etiology of community-acquired pneumonia in hospitalized children based on WHO clinical guidelines. Eur J Pediatr. 2009;168:1429-1436.

10. Lim I, Shaw DR, Stanley DP, et al. A prospective hospital study of the aetiology of community-acquired pneumonia. Med J Aust. 1989;151:87-91.

11. Charles PG, Whitby M, Fuller AJ, et al. The etiology of communityacquired pneumonia in Australia: why penicillin plus doxycycline or a macrolide is the most appropriate therapy. Clin Infect Dis. 2008;46:15131521.

12. National Health and Medical Research Council. The Australian Immunisation Handbook. Canberra, Australia: Australian Government Department of Health and Aging; 2008

13. Black SB, Shinefield HR, Ling S, et al. Effectiveness of heptavalent pneumococcal conjugate vaccine in children younger than five years of age for prevention of pneumonia. Pediatr Infect Dis J. 2002;21:810-815.

14. Klugman KP, Madhi SA, Huebner RE, et al. A trial of a 9-valent pneumococcal conjugate vaccine in children with and those without HIV infection. N Engl J Med. 2003;349:1341-1348.

15. Cutts FT, Zaman SM, Enwere G, et al. Efficacy of nine-valent pneumococcal conjugate vaccine against pneumonia and invasive pneumococcal disease in The Gambia: randomised, double-blind, placebo-controlled trial. Lancet. 2005;365:1139-1146.

16. Mahon BE, Hsu K, Karumuri S, et al. Effectiveness of abbreviated and delayed 7-valent pneumococcal conjugate vaccine dosing regimens. Vaccine. 2006;24:2514-2520. 
17. Whitney CG, Pilishvili T, Farley MM, et al. Effectiveness of seven-valent pneumococcal conjugate vaccine against invasive pneumococcal disease: a matched case-control study. Lancet. 2006;368:1495-1502.

18. Grijalva CG, Nuorti JP, Arbogast PG, et al. Decline in pneumonia admissions after routine childhood immunisation with pneumococcal conjugate vaccine in the USA: a time-series analysis. Lancet. 2007;369:1179-1186.

19. Zhou F, Kyaw MH, Shefer A, et al. Health care utilization for pneumonia in young children after routine pneumococcal conjugate vaccine use in the United States. Arch Pediatr Adolesc Med. 2007;161:1162-1168.

20. Grijalva CG, Griffin MR, Nuorti JP, et al. Pneumonia hospitalizations among young children before and after introduction of pneumococcal conjugate vaccine-United States, 1997-2006. MMWR Morb Mortal Wkly Rep. 2009; $58: 1-4$.

21. Nelson JC, Jackson M, Yu O, et al. Impact of the introduction of pneumococcal conjugate vaccine on rates of community acquired pneumonia in children and adults. Vaccine. 2008;26:4947-4954.

22. De Wals P, Robin E, Fortin E, et al. Pneumonia after implementation of the pneumococcal conjugate vaccine program in the province of Quebec, Canada. Pediatr Infect Dis J. 2008;27:963-968.

23. National Centre for Classification in Health. International Classification of Diseases, 10th revision, Australian Modification (ICD-10-AM). 2nd ed. Sydney, Australia: National Centre for Classification in Health; 2000.

24. Marrie TJ. Pneumococcal pneumonia: epidemiology and clinical features. Semin Respir Infect. 1999;14:227-236.

25. Elliott JH, Anstey NM, Jacups SP, et al. Community-acquired pneumonia in northern Australia: low mortality in a tropical region using locallydeveloped treatment guidelines. Int J Infect Dis. 2005;9:15-20.

26. Thompson JE. Community acquired pneumonia in north eastern Australia-a hospital based study of Aboriginal and non-Aboriginal patients. Intern Med J. 1997;27:59-61.

27. Australian Institute of Health and Welfare. Improving the quality of indigenous identification in hospital separations data. AIHW cat. no. HSE 101 (Health Services Series no. 25). Canberra, Australia: AIHW; 2005.

28. Brotherton J, Wang H, Schaffer A, et al. Vaccine preventable diseases and vaccination coverage in Australia, 2003 to 2005. Commun Dis Intell. 2007;31(suppl):S1-S152.

29. Lopalco P, Editorial team. Use of 7-valent pneumococcal conjugate vaccine in EU. Euro Surveill. 2006;11: 3092. Available at: http:// www.eurosurveillance.org/ViewArticle.aspx?ArticleId=3092. Accessed June 19, 2008.

30. Committee on Infectious Diseases. Recommended immunization schedules for children and adolescents--United States, 2007. Pediatrics. 2007;119: 207-208.
31. Millar EV, O'Brien KL, Bronsdon MA, et al. Anticapsular serum antibody concentration and protection against pneumococcal colonization among children vaccinated with 7 -valent pneumococcal conjugate vaccine. Clin Infect Dis. 2007;44:1173-1179.

32. Dagan R, Givon-Lavi N, Fraser D, et al. Serum serotype-specific pneumococcal anticapsular immunoglobulin g concentrations after immunization with a 9-valent conjugate pneumococcal vaccine correlate with nasopharyngeal acquisition of pneumococcus. J Infect Dis. 2005;192:367-376.

33. Jardine A, Menzies RI, Deeks SL, et al. The impact of pneumococcal conjugate vaccine on rates of myringotomy with ventilation tube insertion in Australia. Pediatr Infect Dis J. 2009;28:761-765.

34. Menzies R, Turnour C, Chiu C, et al. Vaccine preventable diseases and vaccination coverage in Aboriginal and Torres Strait Islander people, Australia 2003 to 2006. Commun Dis Intell. 2008;32(suppl):S2-S67.

35. Boulianne N, De Wals $P$, Deceuninck G, et al. Impact du programme d'immunisation par le vaccine pneumococcique conjugue heptavelent (VPC-7), au Quebec, Canada. Institut National de Sante Publique de Quebec, 2007. Available at: http://www.inspq.qc.ca/publications/notice.asp? $\mathrm{E}=\mathrm{p} \&$ NumPublication=681. Accessed May 20, 2009.

36. Darling N, Kolasa M, Wooten KG. National, state, and local area vaccination coverage among children aged 19-35 months--United States, 2007. MMWR Morb Mortal Wkly Rep. 2008;57:961-966.

37. Fine MJ, Auble TE, Yealy DM, et al. A prediction rule to identify low-risk patients with community-acquired pneumonia. $N$ Engl J Med. 1997;336: 243-250.

38. Lee RW, Lindstrom ST. A teaching hospital's experience applying the Pneumonia Severity Index and antibiotic guidelines in the management of community-acquired pneumonia. Respirology. 2007;12:754-758.

39. Maxwell DJ, McIntosh KA, Pulver LK, et al. Empiric management of community-acquired pneumonia in Australian emergency departments. Med J Aust. 2005;183:520-524.

40. Isaacs D. Problems in determining the etiology of community-acquired childhood pneumonia. Pediatr Infect Dis J. 1989;8:143-148.

41. Skull SA, Andrews RM, Byrnes GB, et al. ICD-10 codes are a valid tool for identification of pneumonia in hospitalized patients aged $\geq 65$ years. Epidemiol Infect. 2008;136:232-240.

42. World Health Organization. Pneumococcal conjugate vaccine for childhood immunization - -WHO position paper. Wkly Epidemiol Rec. 2007;82:93-104.

43. Sinha A, Levine O, Knoll MD, et al. Cost-effectiveness of pneumococcal conjugate vaccination in the prevention of child mortality: an international economic analysis. Lancet. 2007;369:389-396.

44. Ramsay ME, McVernon J, Andrews NJ, et al. Estimating Haemophilus influenzae type $\mathrm{b}$ vaccine effectiveness in England and Wales by use of the screening method. J Infect Dis. 2003;188:481-485. 